\title{
Review
}

Andrea Vianello*, Gabriella Guarnieri, Fausto Braccioni, Sara Lococo, Beatrice Molena, Antonella Cecchetto, Chiara Giraudo, Leonardo Bertagna De Marchi, Marco Caminati and Gianenrico Senna

\section{The pathogenesis, epidemiology and biomarkers of susceptibility of pulmonary fibrosis in COVID-19 survivors}

https://doi.org/10.1515/cclm-2021-1021

Received September 17, 2021; accepted November 8, 2021; published online November 16, 2021

Abstract: Pulmonary fibrosis (PF), a pathological outcome of chronic and acute interstitial lung diseases associated to compromised wound healing, is a key component of the "post-acute COVID-19 syndrome" that may severely complicate patients' clinical course. Although inconclusive, available data suggest that more than a third of hospitalized COVID-19 patients develop lung fibrotic abnormalities after their discharge from hospital. The pathogenesis of PF in patients recovering from a severe acute case of COVID-19 is complex, and several hypotheses have been formulated to explain its development. An analysis of the data that is presently available suggests that biomarkers of susceptibility could help to identify subjects with increased probability of developing PF and may represent a means to personalize the management of COVID-19's long-term effects. Our review highlights the importance of both patient-related and disease-related contributing risk factors for PF in COVID-19 survivors and makes it definitely clear the possible use of acute phase and follow-up biomarkers for identifying the patients at greatest risk of developing this disease.

\footnotetext{
*Corresponding author: Andrea Vianello, Department of Cardiac Thoracic Vascular Sciences and Public Health, UOC Fisiopatologia Respiratoria, Ospedale-Università di Padova, Via Giustiniani, 235128 Padova, Italy, Phone: +0039 049 8218587, Fax: +0039 049 8218590, E-mail: andrea.vianello@aopd.veneto.it. https://orcid.org/00000002-8790-6029

Gabriella Guarnieri, Fausto Braccioni, Sara Lococo, Beatrice Molena, Antonella Cecchetto and Leonardo Bertagna De Marchi, Department of Cardiac Thoracic Vascular Sciences and Public Health, University of Padova, Padova, Italy

Chiara Giraudo, Department of Medicine DIMED, University of Padova, Padova, Italy

Marco Caminati and Gianenrico Senna, Asthma Center and Allergy Unit, University of Verona, Verona, Italy
}

Keywords: ACE2; biomarker; COVID-19; interleukin; pulmonary fibrosis.

\section{Introduction: the long-term health consequences of COVID-19}

On December 31st, 2019 the WHO China Country Office was informed of cases of pneumonia of unknown etiology detected in Wuhan City; the report signaled the beginning of the coronavirus disease 2019 (COVID-19) emergency and pandemic, with currently over 183 million confirmed cases and four million deaths recorded [1]. As the pandemic evolves, evidence-based medical treatments including anticoagulants, corticosteroids, oxygen therapy and mechanical ventilation (MV) have succeeded in improving patients' outcomes, and the majority are now expected to recover spontaneously or after acute phase management [2]. In particular, a prolonged administration of low-dose methylprednisolone or dexamethasone has been found to reduce mortality in hospitalized patients with severe disease [3, 4]; accordingly, a WHO guideline panel has recommended systemic corticosteroids in patients with severe or critical COVID-19 [5].

While effective treatments for the acute phase were feverishly being developed, the scientific community also set out to investigate the clinical and functional long-term consequences of COVID-19, which include a complexity of symptoms and organ-related injuries referred to as "long COVID” or "post-acute COVID-19 syndrome" (PACS) [6]. The term "post-acute COVID-19 syndrome" has been proposed to refer to the symptoms and/or abnormalities not attributable to another cause persisting or present beyond four weeks from the time the disease was diagnosed [7]. The severity and extent of the long-term effects of COVID-19 are even now not entirely clear: indeed, on April 1, 2021, the Office for National Statistics estimated that 1.1 million people in the UK were experiencing PACS [8]. 
According to the scientific literature, PACS is a heterogeneous condition, consisting of a constellation of approximately 50 symptoms, including fatigue, headache, breathlessness, chest discomfort, attention disorders, neurological symptoms such as dementia, depression, anxiety, as well as sleep disturbances and organ lesions affecting the respiratory, cardiovascular, renal, endocrine, ear-nose-throat, gastrointestinal or dermatological systems, and many patients have reported experiencing multiple, overlapping symptoms (Figure 1) [9]. Considered a key component of PACS, pulmonary fibrosis (PF), can severely complicate the clinical course of COVID-19 survivors. Indeed, almost half of patients with moderate to severe COVID-19 pneumonia may show impaired pulmonary diffusion after discharge from hospital. Moreover, lung fibrotic changes on chest CT scan have been reported in approximately one third of patients who recovered from severe or critical disease. It appears that residual fibrotic lesions could be prevented by the prolonged use of low-dose corticosteroids [10, 11].

The current review focuses on the epidemiology and pathogenesis of post-COVID-19 PF and the biomarkers that may be able to identify the patients who have an increased likelihood of developing the disease. In particular, this study aims to describe different pathogenic hypotheses formulated to explain the development of PF and investigate both the acute phase and follow-up biomarkers that may predict the onset of pulmonary fibrotic lesions.

\section{Pulmonary fibrosis consequent to COVID-19: epidemiology and risk factors}

A pathological outcome of acute/chronic interstitial lung diseases associated with abnormal wound healing, PF may contribute to alterations in lung mechanics and pulmonary gas exchange. The most important histopathological characteristics of the disease are: impaired reconstruction of the damaged alveolar epithelium, persistence of fibroblasts, excessive deposition of extracellular matrix (ECM) components such as collagen, and the destruction of normal lung architecture [12]. Notably, clinical, radiographic, and autopsy findings of PF were commonly noted in patients with severe acute respiratory syndrome (SARS) and Middle East respiratory syndrome (MERS), conditions caused by a coronavirus similar to COVID-19.

As the number of patients recovering from severe COVID-19 disease rises, it is becoming increasingly apparent that a substantial number show signs of postinflammatory PF, in particular those patients overcoming COVID-19 related Acute Respiratory Distress Syndrome (ARDS) requiring treatment with high flow nasal oxygen (HFNO) and/or MV [13, 14]. Indeed, PF subsequent to ARDS is a well-recognized phenomenon. In fact, when Thille et al. [15] analyzed 169 autopsies of patients with ARDS linked to a variety of etiologies, they reported that PF was present in three $(4 \%)$ out of 82 patients with disease

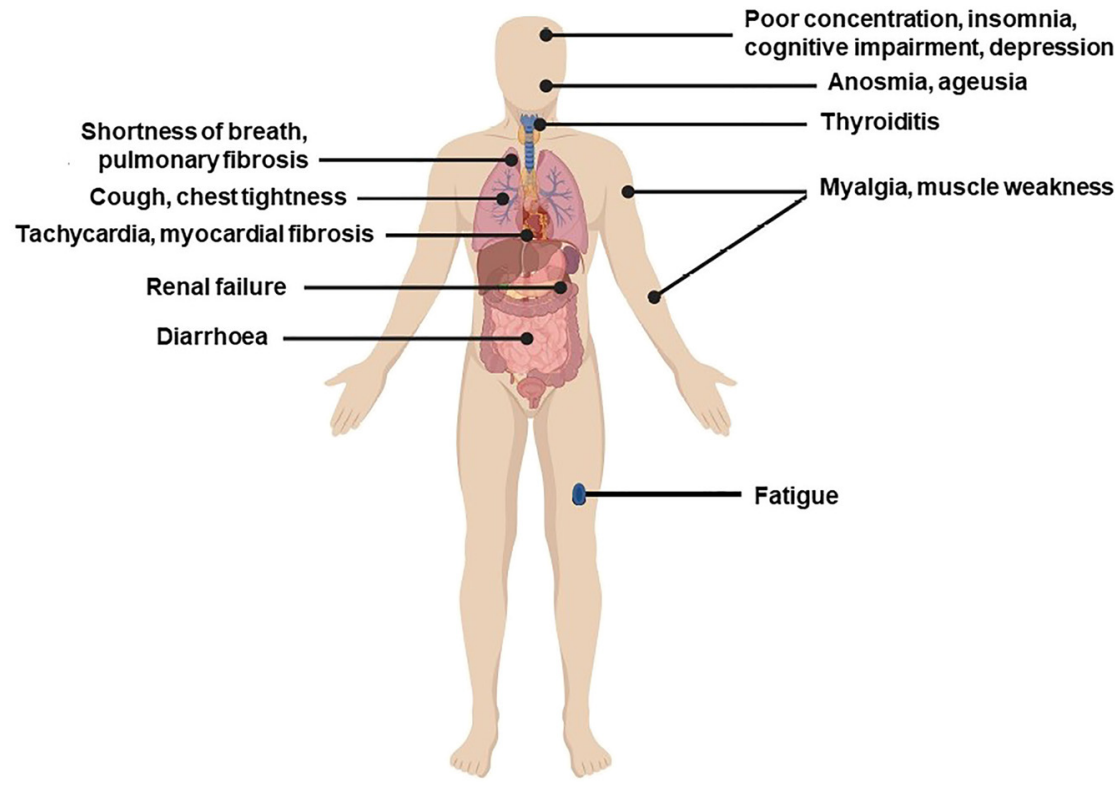

Figure 1: Clinical manifestations of patients with "post-acute COVID-19 syndrome", indicating the possible involvement of the respiratory, cardiovascular, nervous, renal, endocrine, ear-nose-throat, gastrointestinal, musculoskeletal and dermatological system. 
duration of less than 1 week, 13 (24\%) out of 54 patients with 1 to 3-week disease duration, and 14 (61\%) of 23 patients with disease duration longer than three weeks. Moreover, when Desai et al. analyzed 27 patients who underwent MV to treat ARDS, they reported that 23 (85\%) showed signs of PF 110-267 days after extubation, and a close association with the duration of the pressurecontrolled inverse-ratio ventilation was noted [16].

Since $7.2-31 \%$ of COVID-19 patients suffer from viral induced ARDS [17], PF can be considered an important long-term adverse health outcome of the disease [18]. Indeed, bilateral, extensive lung involvement and histopathological findings of diffuse alveolar damage and fibromyxoid cell exudates have been considered structural factors predisposing to impaired lung reconstruction in patients who have overcome a severe case of COVID-19 [19]. Of interest, patients with severe COVID-19 leading to ARDS may also show full thickness tracheal/large airway cartilage lesions with fibrous-hyaline degeneration: this pathologic feature is uncommon in individuals with nonCOVID ARDS and could have contributed to the unexpectedly high prevalence of pneumomediastinum reported during the second wave of COVID-19 pandemic [20, 21].

A diagnosis of post-COVID-19 PF should be based on clinical, radiologic, and pathologic findings. While the appropriate timing for a diagnosis of irreversible PF has not yet been established, many specialists recommend serial lung function tests, chest CT scans and exercise tests 3, 6, and 12 months after the onset of an acute COVID-19 episode [12]. Due to the diagnostic challenges linked to the current pandemic, there are no reliable data on the frequency of $\mathrm{PF}$ complicating COVID-19. Nevertheless, data collected at the time of hospital discharge suggest that more than a third of COVID-19 patients may develop fibrotic abnormalities [22]. $\mathrm{Hu}$ et al. reported that reticular changes, which may represent a sign of irreversible fibrosis, were evident on chest CT scan in 23 out of 46 patients two weeks after the onset of the disease and persisted at the four-week followup [23]. Similarly, Pan et al. reported fibrotic alterations on the chest CT scans of 11 out of 63 patients during the acute phase [24]. When Huang et al. carried out a longitudinal analysis of CT findings in patients hospitalized for COVID-19 two months after discharge, they found that 52\% (42/81) showed extensive fibrosis [25]. Finally, Wallis et al. reported that 32 out of 101 patients had persistent chest X-ray (CXR) abnormalities at their 12-week post hospitalization follow-up. Notably, the length of the patients' hospital stay, smoking-status and obesity were found to be independent risk factors for persistent abnormalities. Moreover, serum lactate dehydrogenase (LDH) levels were significantly higher both at baseline and follow-up testing times in the patients with abnormalities compared to those who had recovered [26].

It is important that clinicians are able to recognize potential contributing risk factors for PF. Depending on their origin, these can be classified as patient-related or disease-related (Table 1). Some studies have demonstrated that patients at higher risk of PF tend to be older, male, active smokers and have underlying disorders, such as diabetes and lung and cardiovascular diseases. Indeed, lung fibrosis due to a variety of etiologies is more frequently found in elderly adults. Accordingly, a 230-day follow-up study uncovered that older patients were more likely to develop PF following MERS [27]. Active smoking has been associated to the pathogenesis of various lung diseases such as emphysema, chronic bronchitis, and PF. Epidemiological studies have shown a higher incidence of familial and sporadic Idiopathic PF (IPF) in smokers with respect to their nonsmoking counterparts. A multivariable logistic analysis by Liu et al. uncovered that current smoking was a factor determining progression of lung fibrosis in a group of 78 COVID-19 patients [28]. It has also been suggested that chronic alcoholism increases the risk of PF in COVID-19 patients [29].

Comorbidities also seem to play a decisive role in the development of post COVID-19 PF. Huang et al. used an observational cohort study design to investigate potential indicators of post-COVID-19 PF. Eighty-one COVID-19 survivors who had undergone at least three follow-up CT scans after discharge from hospital were divided into two groups: a fibrosis $(n=42)$ one and non-fibrosis $(n=39)$ one depending on the presence/absence of extensive, persistent fibrotic changes on the CT scans. Compared to the non-

Table 1: Potential contributing risk factors for pulmonary fibrosis in COVID-19 survivors.

\begin{tabular}{ll}
\hline Patient-related risk factors & $\begin{array}{l}\text { Disease-related risk } \\
\text { factors }\end{array}$ \\
\hline Male gender & $\begin{array}{l}\text { Length of hospital stay } \\
\text { Length of ICU stay } \\
\text { Older age }\end{array}$ \\
Active smoking & Presence of ARDS \\
Alcohol abuse & Degree of systemic \\
Comorbidities: & inflammation \\
- Diabetes & \\
- Obesity & \\
$-\quad$ Hypertension & \\
$-\quad$ Chronic pulmonary disease & \\
$-\quad$ Chronic liver disease & \\
$-\quad$ Cardiovascular and cerebrovascular & \\
\hline
\end{tabular}

ARDS, acute respiratory distress syndrome; HFNO, high flow nasal oxygen; ICU, intensive care unit; MV, mechanical ventilation. 
fibrotic patients, the fibrotic ones were older $(p<0.001)$, disproportionately male $(\mathrm{p}=0.036)$, had more underlying diseases ( 78 vs. $41 \%$ ), including diabetes (31 vs. $23 \%$ ), hypertension ( 40 vs. $23 \%$ ), chronic pulmonary disease (21 vs. $7 \%$ ), chronic liver disease (19 vs. $13 \%$ ), cardiovascular and cerebrovascular disease ( 29 vs. $15 \%$ ) [25].

It has also been noted that the length of stay in a normal ward and in an Intensive Care Unit (ICU), the use of HFNO, the need for MV, and the presence of ARDS seem to be associated with a higher risk of PF [30, 31]. Several clinicians have postulated that prolonged MV-induced lung injury (VILI), which consists in an acute lung injury arising from or exacerbated by MV and is associated with increased ICU mortality in ARDS, may play a role in the development of PF [32]. Abnormalities in ventilator pressure and/or volume settings may underlie the injury leading to a release of pro-inflammatory modulators, worsening acute lung injury and increased mortality or PF in survivors [33].

Severe systemic inflammation may also predict the onset of post-COVID-19 PF. Huang et al. demonstrated that neutrophil, the neutrophil-lymphocyte ratio (NLR), C-reactive protein (CRP) and LDH levels were markedly above the normal range during the first four weeks following hospital admission in patients showing extensive fibrosis at the two month follow-up. Conversely, CRP and LDH increased within two weeks, but then sharply dropped to normal levels in the non-fibrosis group [25].

\section{The pathogenesis of post-COVID-19 pulmonary fibrosis}

Following an initial phase of lung injury causing acute inflammation, repair mechanisms can elicit the restoration of normal pulmonary architecture or they may lead to PF with architectural distortion and irreversible lung dysfunction. In normal conditions, during the early stages of lung injury, acute damage to the endothelial cells leads to the release of inflammatory mediators and triggers an antifibrinolytic coagulation cascade that temporarily plugs the damaged vessel with a platelet and fibrin-rich clot [34]. Platelet recruitment, degranulation and clot formation rapidly lead to vasodilation with increased permeability, allowing extravasation and the direct recruitment of leukocytes to the injured site. Subsequently, access to the site of tissue damage is facilitated by the action of zinc-dependent endopeptidases, also called matrixmetalloproteinases (MMPs), which may disrupt the basement membrane by cleaving one or more ECM constituents. As a consequence, chemokine gradients may recruit inflammatory cells. Neutrophils, eosinophils, and lymphocytes are present at the sites of acute lung injury with cell debris and areas of necrosis cleared by alveolar macrophages [35]. The nature of the inflammatory response may influence the resident tissue cells and the ensuing inflammatory cells, since the latter additionally exacerbate inflammation by secreting chemokines, cytokines, and growth factors. Many cytokines are involved throughout the woundhealing response, with specific groups of genes activated in different conditions. Interleukin (IL)-4 (IL-4), IL-13, and transforming growth factor-beta (TGF- $\beta$ ) are cytokines that have received attention with regard to various pulmonary fibrotic conditions since each can exhibit pro-fibrotic activity by promoting the recruitment, activation and proliferation of fibroblasts, macrophages, and myofibroblasts [36]. The final phase of wound healing consists of tissue repair, involving angiogenesis, fibroblast activation, and collagen deposition. In the presence of alveolar exudates, organization occurs which consists in the fibroblastic invasion of the alveoli and transformation into myofibroblasts leading to the deposition of the ECM. Epidermal growth factor (EGF) and transforming growth factor-alpha (TGF- $\alpha$ ) stimulate proliferation of bronchiolar stem cells to replace damaged alveolar epithelium. Finally, vascular endothelial growth factor (VEGF) and fibroblast growth factor (FGF) stimulate the migration and proliferation of uninjured endothelial cells leading to pulmonary capillary angiogenesis [37]. In the event of ARDS, a condition characterized by persistent injury with damage to the basement membranes, fibroblastic activities may instead persist, converting organizing into fixed and/or progressive fibroblastic tissue. As a consequence, the formation of either focal or diffuse scar tissue may result in disorganized alveolar architecture, and excessive deposition of ECM is a central aspect of the process [38].The pathogenesis of $\mathrm{PF}$ in patients overcoming a severe case of COVID-19 is complex, and several hypotheses (listed below) have been formulated to explain its development (Figure 2).

\section{Virally activated profibrotic pathway}

Structural analysis has uncovered that residues in the receptor binding domain (RBD) of SARS-CoV-2 have a high affinity to angiotensin-converting enzyme 2 (ACE2), a receptor expressed in the airway and alveolar epithelial cells consisting of 805 amino acids, acting as a regulator of the renin-angiotensin system (RAS). Through ACE2-mediated endocytosis, SARS-CoV-2 endocytosed into epithelial cells is released and undergoes rapid replication, leading to 


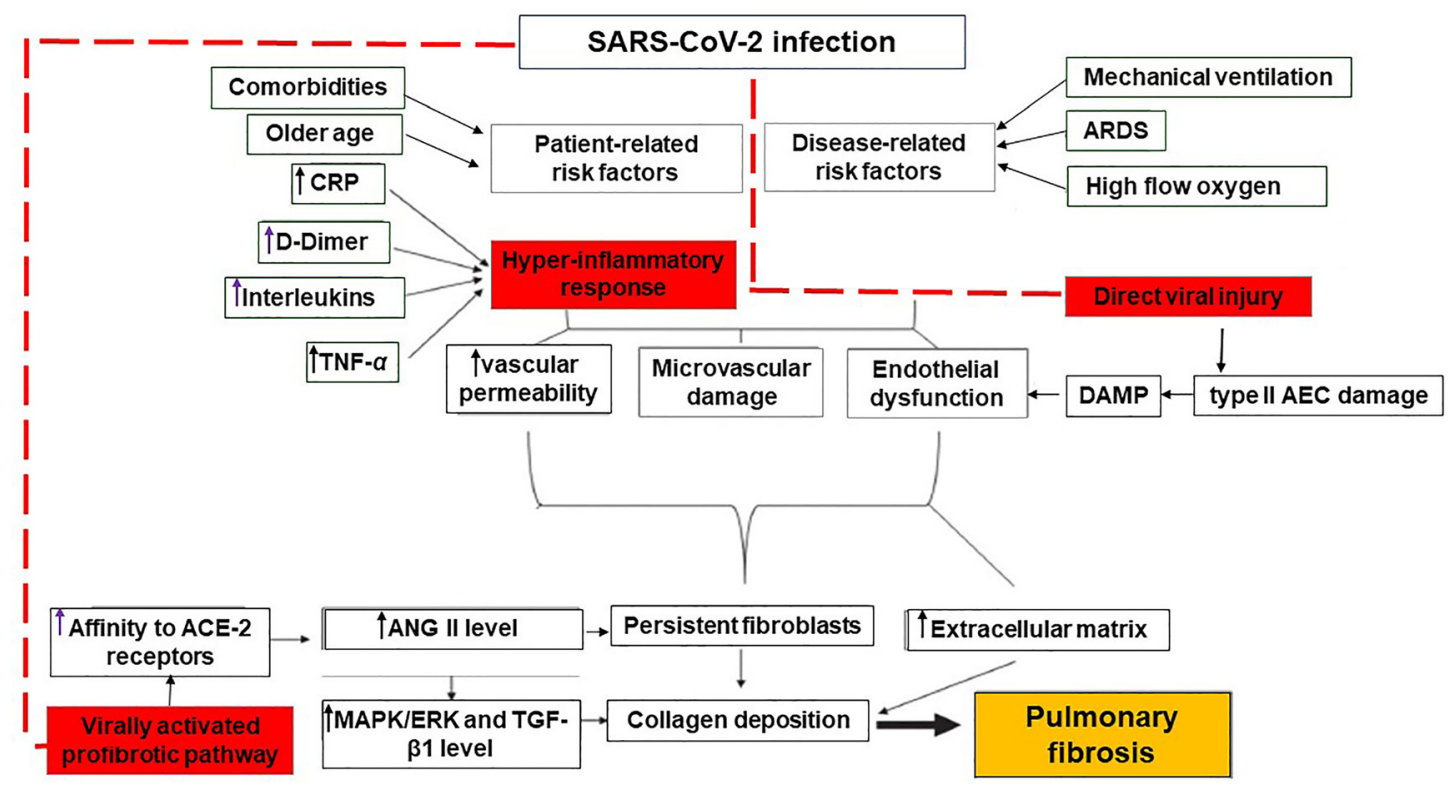

Figure 2: Pathophysiological pathways underlying pulmonary fibrosis in COVID-19 survivors.

Multiple cellular and molecular mechanisms may contribute to the pathogenesis of the disease, including direct viral and indirect patientspecific immune-mediated damage (ACE2, angiotensin-converting enzyme 2; AEC, alveolar epithelial cells; ANG II, angiotensin II; ARDS, acute respiratory distress syndrome; CRP, C-reactive protein; DAMPs, damage-associated molecular patterns; ERK, extracellular signal-regulated kinase; MAPK, mitogen-activated protein kinase; TGF- $\beta 1$, transforming growth factor-beta 1 ; TNF- $\alpha$, tumor necrosis factor-alpha).

pyroptosis, a typical virus-linked programmed cell death. A significant downregulation of ACE2 expression occurs due to ACE2-mediated endocytosis, which reduces the host's ability to balance RAS. Since ACE2 functions as a counter-regulator of RAS, the decrease in ACE2 expression leads to a weakened ACE2-Ang (1e7)-MasR axis, primarily manifested in a higher angiotensin II (Ang II) level and a lower vasodilator Ang (1e7) level; the latter may induce protective anti-inflammatory and antifibrotic effects in normal conditions [39]. The finding that plasma Ang II levels in 12 COVID-19 patients were significantly higher than those in uninfected individuals supports this hypothesis [40]. As a consequence, Ang II, which activates a broad range of signaling pathways, seems to increase its proinflammatory and profibrotic effects. While the former effects include activation of IL-6, tumor necrosis factor-alpha (TNF- $\alpha$ ), and increased recruitment of neutrophils and macrophages as well as direct endothelial cell injury, the latter involve promoting collagen I gene activation through MAPK/ERK and TGF- $\beta$, both critical factors in the fibrotic response [18]. It has been hypothesized that $\alpha 1$-Antitrypsin (AAT) may hinder SARS-CoV-2 cell entry after ACE2 binding by inhibition of TMPRSS2-mediated priming and that its deficiency may play a central role in the pathogenicity and virulence of SARS-CoV-2 [41-43].

\section{Direct viral injury}

Although still unconfirmed, some have hypothesized that a viral injury itself may play a key role in the development of PF, resulting in an altered cell function and secretory profile as well as cell death. Despite the fact that multiple cell types may become infected, the infection and death of type II alveolar epithelial cells (AEC) seem to be of critical importance as they stabilize and repair the epithelial barrier, secrete vital prosurfactant and are not efficiently replaced [44, 45]. Following an injurious stimulus, AEC may release damage-associated molecular patterns (DAMPs), which, together with pathogen-associated molecular patterns (PAMPs) from microbes, are recognized by alveolar macrophages, leading to a series of downstream transduction and release of antimicrobial and proinflammatory cytokines, including IL- 1 and TNF- $\alpha$. In the light of these considerations, type II AEC damage induced by viral infection is thought to be an initiating event triggering the development of PF [46]. At the same time, the disruption of the alveolar epithelial surface and the loss of 
alveolar macrophage populations can leave endothelial cells susceptible to direct infection. Endothelial cell death is important since it leads to the disruption of barrier function and higher vascular permeability which substantially increases the extent of pulmonary injury. This type of damage to the lung microvascular architecture may also contribute to PF development [47].

\section{Hyper-inflammatory response}

Other potential determinants of post-COVID-19 PF include a hyper-inflammatory response to the virus leading to the activation of a complex cytokine pathway. Indeed, the SARS-CoV-2 infection may affect the inflammatory response, inducing a specific inflammatory pattern unlike the ones during other viral infections. In normal conditions, cytokines and chemokines play an important role in the wound-healing response by promoting activation of immune populations that clear infection via $\mathrm{T}$ cell and B cell recruitment. They also lead to the activation of macrophage populations that efficiently clear apoptotic cellular debris. An immune hyper-reaction referred to as a "cytokine storm" may, instead, occur in patients with severe COVID-19 and lead to an uncontrolled release of an excessive amount of cytokines, including IL-1- $\beta$, IL-6, IL-7, IL-8, IL-9, IL-10, monocyte chemoattractant protein, and TNF- $\alpha$. "Cytokine storms" can cause apoptosis of endothelial and epithelial cells, ischemia, and hypoxia, leading to inflammatory-induced lung injury, ARDS and multiorgan failure resulting in poor patient outcome and even death [48]. If the "cytokine storm" is not interrupted in a timely manner, it can also result in lung tissue damage, stiffening and altered vascularization, and finally PF. It has been found that the balance of chemokines and cytokines vs. interferons is essential to eliminating infection without inducing significant lung injury [49].

\section{Mechanical injury}

Since MV applies physical stresses to the tissues of the lung, it may give rise to VILI, particularly in patients with ARDS. Increased lung stretch can induce oxidative injury, stimulate cytokine production and epithelial-mesenchymal transition (EMT) and finally increase collagen deposition in the lungs [50]. Yang et al. [51] demonstrated in a murine acid aspiration model that the expression of EMT markers is associated to PF development.

\section{Susceptibility biomarkers to identify patients at risk of developing pulmonary fibrosis}

Since it has been demonstrated that PF consequent to ARDS may lead to high levels of morbidity and mortality, it is crucial to identify the COVID-19 patients at risk of developing fibrotic damage permitting timely action to minimize morpho-functional damage to the lungs. Since no reliable clinical or laboratory indicators are as yet available during the early phases of disease progression, some have proposed using susceptibility biomarkers to identify subjects with an increased probability of developing PF. The acute and follow-up phase biomarkers that have been identified until now are listed in Table 2.

\section{Risk biomarkers during acute COVID-19}

Higher CRP and lower lymphocyte count are the laboratory parameters that have been considered potential risk factors for fibrotic changes during an acute phase of COVID-19. According to a retrospective study performed by $\mathrm{Yu}$ et al. involving 32 confirmed COVID-19 patients who were classified depending on signs or no signs of fibrosis on their latest follow-up CT imaging, the two groups had significantly different CRP levels $(53.4 \mathrm{mg} / \mathrm{L}$ vs. $10.0 \mathrm{mg} / \mathrm{L}, \mathrm{p}=0.002)$ and lymphocyte count [0.6 (0.4-0.9) vs. $1.1(0.8-1.3)$; $\mathrm{p}=0.003$ ] [52]. Huang et al.'s [25] observational cohort study comparing fibrosis/non-fibrosis groups reported that CRP and LDH levels were markedly above normal for four

Table 2: Potential susceptibility biomarkers to identify patients at risk of developing post-COVID-19 pulmonary fibrosis.

\begin{tabular}{ll}
\hline Acute COVID-19 & Follow-up period \\
\hline C-reactive protein & TNF- $\alpha$ \\
Lymphocyte count & IL-17A, IL-17D \\
LDH & VCAM-1, ICAM-1, PIGF \\
IFN- $\gamma$ & KL-6 \\
MMP-9 & \\
SST2 & \\
\hline
\end{tabular}

ICAM-1, intercellular adhesion molecule-1; IL-17, interleukin-17; IFN- $\gamma$, interferon-gamma; LDH, lactate dehydrogenase; MMP-9, metalloproteinase-9; PIGF, placental growth factor; SST2, soluble suppressor of tumorigenicity 2; VCAM-1, vascular cell adhesion molecule-1. 
consecutive weeks during the acute phase and that higher CRP levels persisted after that date in the fibrosis group.

Yan et al. [53] retrospectively analyzed the blood samples of 485 patients in the attempt to identify markers of mortality risk. Using a mathematical modeling approach, the authors identified three prognostic indicators of COVID-19 (LDH, CRP and lymphocytes). In particular, elevated LDH reflects tissue destruction and was regarded as a prognostic marker of lung injury.

When $\mathrm{Hu}$ et al. [49] investigated the effect of a series of inflammatory mediators in 76 hospitalized COVID-19 patients, they uncovered that fibrosis was present in the 46 $(60.5 \%)$ whose plasma interferon-y (IFN-y) levels were twofold lower than those without fibrosis ( $p>0.05)$. The finding led them to conclude that a mild interferon response may be a risk factor for $\mathrm{PF}$.

Ueland et al. [54] investigated plasma biomarkers in 39 patients with SARS-CoV-2 infection within $48 \mathrm{~h}$ of hospitalization in an attempt to identify an association between biomarker temporal profile and acute respiratory failure (ARF). Although, multiple markers were associated with ARF, expressed by $\mathrm{P} / \mathrm{F}$ ratio, the association between $\mathrm{P} / \mathrm{F}$ ratio and metalloproteinase (MMP)-9 profile was the only significant one after adjusting for confounding factors, and MMP-9 distinguished patients with and without ARF. The study's findings suggest that MMP-9 may be an early indicator of the risk of respiratory failure in COVID-19 patients and they highlight the role of ECM remodeling in patients' progression to PF. That conclusion is supported by other data showing that zinc-dependent proteases MMP-1 and MMP-7, which are involved in the breakdown and the remodeling of ECM components, are overexpressed in the plasma of IPF patients with respect to hypersensitivity pneumonitis, sarcoidosis and chronic obstructive pulmonary disease (COPD) subjects [55].

Some studies have recently shown that soluble suppressor of tumorigenicity 2 (sST2), which is a member of IL-1 receptor family routinely used as prognostic biomarker in patients with heart failure, may be a risk biomarker for PF progression. High circulating SST2 levels have in fact been found in subjects with ARDS, PF and sepsis. The SST2 ligand is IL-33, a member of the IL-1 cytokine family secreted by several cell types following injury. It has been suggested that the IL-33/ST2 axis may be involved in the development of PF since in vivo studies have demonstrated that IL-33 expression levels increased in parallel with the development of PF in mice undergoing bleomycin treatment [56]. Ragusa et al. hypothesized that circulating sST2 levels can be used as a risk biomarker to predict the onset of $\mathrm{PF}$ after discharge in COVID-19 patients [57].

\section{Risk biomarkers during follow-up}

A number of biomarkers potentially reflecting progression in post COVID-19 PF have recently been evaluated. CT and pulmonary function assessment tests, multiplex cytokine assay, flow cytometry and 44 soluble markers were investigated by Zhou et al. who studied 72 individuals, including COVID-19 patients discharged from four hospitals in Wuhan, recovered asymptomatic patients (APs) from an isolation hotel, and uninfected healthy controls (HCs) [58]. The participants' plasma cytokines were categorized into four classes: Class 1: cytokines associated with vascular injury and repair/angiogenesis; Class 2: factors promoting immune cell growth and differentiation; Class 3: proinflammatory immune factors; Class 4: chemokines. Three months after discharge, the levels of pro-inflammatory cytokines, in particular TNF- $\alpha$ IL-17A and IL-17D and factors related to vascular injury/repair, in particular, vascular cell adhesion molecule-1 (VCAM-1), intercellular adhesion molecule-1 (ICAM-1), and placental growth factor (PIGF) were significantly higher in the recovered COVID-19 patients and in particular in those who had a severe case than in their healthy counterparts. Moreover, the class 1, 3, and 4 cytokines were significantly associated with LDH levels, residual CT and pulmonary function test (PFT) abnormalities. The authors thus concluded that aberrant vascular-injury related cytokines, inflammatory factors, and chemokines may predict residual clinical and pulmonary function abnormalities and future complications in recovered Covid-19 patients. Another potential marker of pulmonary epithelial cell injury is Krebs von den Lungen 6 (KL-6), which is a mucin like glycoprotein mainly distributed on the surface of type II AECs and respiratory bronchiolar epithelial cells that has been shown to exert chemotactic and anti-apoptotic effects on fibroblast cells. It has been reported that the levels of KL-6 in the epithelial lining fluid (ELF) of patients with acute lung injury (ALI) and interstitial lung disease are higher with respect to those in control subjects. In addition, elevated serum KL-6 levels have been associated with severe IPF $[59,60]$.

Arnold et al. set out to evaluate KL-6 as a potential marker of the severity of acute COVID-19 infection and/or the persistence of symptoms/radiological abnormalities over a medium term period in 93 convalescing COVID-19 patients invited to systematic clinical follow-up appointments. There was no statistical difference in the baseline KL-6 level between severe and less severe patients evaluated at 28 days. Those authors showed that patients with CT scan abnormalities at 12 weeks had, instead, significantly higher convalescent KL-6 levels compared to the 
remainder of the cohort (1101 vs. $409 \mathrm{IU} / \mathrm{mL}$ ), leading to the conclusion that KL- 6 may help to differentiate patients with persisting dyspnea due to PF from those with deconditioning or dysfunctional breathing alone. Unlike Arnold et al. [61], Peng et al. [62] reported that serum KL-6 concentration on admission was significantly higher in severe patients with COVID-19 compared to those with milder disease. Severe cases displayed a higher incident rate of $\mathrm{PF}$ and more elevated serum KL-6 values at hospital discharge. Unexpectedly, the elevation in serum concentration of fibronectin, an essential component of fibrotic ECM, was not significant.

In the light of the observation that many non-invasive serological biomarkers reflecting tissue remodeling and fibrosis have been shown to predict risk of acute exacerbations, lung function decline and mortality in IPF and other interstitial lung diseases, Leeming et al. suggested using systemic biomarkers of wound healing and ECM remodeling as prognostic markers in COVID-19 patients. More specifically, they proposed using neoepitope technology which utilizes monoclonal antibodies to detect the generation of newly formed epitopes of collagens and other ECM proteins released during inflammation and the progression of fibrosis. Neoepitopes have, in fact, been shown to be associated to PF progression and mortality in patients with IPF [63].

\section{Conclusions}

Much has yet to learn about the long term effects of COVID-19, including post-inflammatory fibrosis. Our review highlights the importance of both patient-related and disease-related contributing risk factors for PF in COVID-19 survivors and makes it definitely clear the possible use of acute phase and follow-up biomarkers for identifying subjects with an increased probability of developing the disease. We expect to see the number of efficient biomarkers to increase as we improve our understanding of the disease's pathophysiology. Biomarkers can be used not only to predict the development of PF and other COVID-19 complications, but can also be utilized to personalize treatment.

Research funding: None declared.

Author contributions: All authors have accepted responsibility for the entire content of this manuscript and approved its submission.

Competing interests: Authors state no conflict of interest. Informed consent: Not applicable.
Ethical approval: The local Institutional Review Board deemed the study exempt from review.

\section{References}

1. WHO. WHO coronavirus (COVID-19) dashboard. Available from: https://covid19.who.int [Accessed 5 Jul 2021].

2. Chalmers JD, Crichton ML, Goeminne PC, Cao B, Humbert M, Shteinberg M, et al. Management of hospitalised adults with coronavirus disease-19 (COVID-19): a European Respiratory Society living guideline. Eur Respir J 2021;57:2100048.

3. Salton F, Confalonieri P, GU M, Santus P, Harari S, Scala R, et al. Prolonged low-dose methylprednisolone in patients with severe COVID-19 Pneumonia. Open Forum Infect Dis 2020;7:ofaa421.

4. The RECOVERY Collaborative Group. Dexamethasone in hospitalized patients with COVID-19. N Engl J Med 2021;384:693-704.

5. Rochwerg B, Agarwal A, Siemieniuk RA, Agoritsas T, Lamontagne F, Askie L, et al. A living WHO guideline on drugs for COVID-19. BMJ 2020;370:m3379.

6. Nalbandian A, Sehgal K, Gupta A, Madhavan MV, McGroder C, Stevens JS, et al. Post-acute COVID-19 syndrome. Nat Med 2021; 27:601-15.

7. Datta SD, Talwar A, Lee JT. A proposed framework and timeline of the spectrum of disease due to SARS-CoV-2 infection: illness beyond acute infection and public health implications. J Am Med Assoc 2020;324:2251-2.

8. Office for National Statistics. Prevalence of ongoing symptoms following coronavirus (COVID-19) infection in the UK. Statistical Bulletin; 2021.

9. Montani D, Savale L, Beurnier A, Colle R, Noël N, Pham T, et al. Multidisciplinary approach for post-acute COVID-19 syndrome: time to break down the walls. Eur Respir J 2021;58:2101090.

10. Safont B, Tarraso J, Rodriguez-Borja E, Fernández-Fabrellas E, Sancho-Chust JN, Molina V, et al. Lung function, radiological findings and biomarkers of fibrogenesis in a cohort of COVID-19 patients six months after hospital discharge. Arch Bronconeumol (Engl Ed). 2021 Sep 3. https://doi.org/10.1016/j.arbres.2021.08. 014 [Epub ahead of print].

11. Baratella E, Ruaro B, Marrocchio C, Starvaggi N, Salton F, Giudici F, et al. Interstitial lung disease at high resolution CT after SARS-CoV2-related acute respiratory distress syndrome according to pulmonary segmental anatomy. J Clin Med 2021;10:3985.

12. Tanni SE, Fabro AT, de Albuquerque A, Machado Ferreira EV, Verrastro CGY, Sawamura MVY, et al. Pulmonary fibrosis secondary to COVID-19: a narrative review. Expet Rev Respir Med 2021;15:791-803.

13. Zumla A, HuiDS AEI, Memish ZA, Maeurer M. Reducing mortality from 2019-nCoV: host-directed therapies should be an option. Lancet 2020;395:e35-6.

14. Arnold DT, Hamilton FW, Milne A, Morley AJ, Viner J, Attwood M, et al. Patient outcomes after hospitalisation with COVID-19 and implications for follow-up: results from a prospective UK cohort. Thorax 2021;76:399-401.

15. Thille AW, Esteban A, Fernández-Segoviano P, Rodriguez JM, Aramburu JA, Vargas-Errázuriz P, et al. Chronology of histological lesions in acute respiratory distress syndrome with diffuse 
alveolar damage: a prospective cohort study of clinical autopsies. Lancet Respir Med 2013;1:395-401.

16. Desai SR, Wells AU, Rubens MB, Evans TW, Hansell DM. Acute respiratory distress syndrome: $\mathrm{CT}$ abnormalities at long-term follow-up. Radiology 1999;210:29-35.

17. Zhou F, Yu T, Du R, Fan G, Liu Y, Liu Z, et al. Clinical course and risk factors for mortality of adult inpatients with COVID-19 in Wuhan, China: a retrospective cohort study. Lancet 2020;395:1054-62.

18. McDonald LT. Healing after COVID-19: are survivors at risk for pulmonary fibrosis? Am J Physiol Lung Cell Mol Physiol 2021;320: L257-65.

19. Borczuk AC, Salvatore SP, SeshanSV, Patel SS, Bussel JB, Mostyka M, et al. COVID-19 pulmonary pathology: a multiinstitutional autopsy cohort from Italy and New York City. Mod Pathol 2020;33:2156-68.

20. Baratella E, Bussani R, Zanconati F, Marrocchio C, Fabiola G, Braga L, et al. Radiological-pathological signatures of patients with COVID-19-related pneumomediastinum: is there a role for the Sonic hedgehog and Wnt5a pathways? ERJ Open Res 2021;7: 00346-2021.

21. Simioli F, Annunziata A, Polistina GE, Coppola A, Di Spirito V, Fiorentino $\mathrm{G}$. The role of high flow nasal cannula in COVID-19 associated pneumomediastinum and pneumothorax. Healthcare (Basel) 2021;9:620.

22. George PM, Wells AU, Jenkins RG. Pulmonary fibrosis and COVID-19: the potential role for antifibrotic therapy. Lancet Respir Med 2020;8:807-15.

23. Hu Q, Guan H, Sun Z, Huang L, Chen C, Ai T, et al. Early CT features and temporal lung changes in COVID-19 pneumonia in Wuhan, China. Eur J Radiol 2020;128:109017.

24. Pan Y, Guan H, Zhou S, Wang Y, Li Q, Zhu T, et al. Initial CT findings and temporal changes in patients with the novel coronavirus pneumonia (2019-nCoV): a study of 63 patients in Wuhan, China. Eur Radiol 2020;30:3306-9.

25. Huang W, Wu Q, Chen Z, Xiong Z, Wang K, Tian J, et al. The potential indicators for pulmonary fibrosis in survivors of severe COVID-19. J Infect 2021;82:e5-7.

26. Wallis TJM, Heiden E, Horno J, Welham B, Burke H, Freeman A, et al. Risk factors for persistent abnormality on chest radiographs at 12-weeks post hospitalisation with PCR confirmed COVID-19. Respir Res 2021;22:157.

27. Das KM, Lee EY, Singh R, Enani MA, Al Dossari K, Van Gorkom K, et al. Follow-up chest radiographic findings in patients with MERS-CoV after recovery. Indian J Radiol Imaging 2017;27:342-9.

28. Liu W, Tao Z-W, Wang L, Yuan M-L, Liu K, Zhou L, et al. Analysis of factors associated with disease outcomes in hospitalized patients with 2019 novel coronavirus disease. Chin Med J (Engl). 2020;133:1032-8.

29. Da BL, Im GY, Schiano TD. COVID-19 hangover: a rising tide of alcohol use disorder and alcohol-associated liver disease. Hepatology 2020;72:1102-8.

30. Guler SA, Ebner L, Beigelman C, Bridevaux P-O, Brutsche M, Clarenbach $C$, et al. Pulmonary function and radiological features four months after COVID-19: first results from the national prospective observational Swiss COVID-19 lung study. Eur Respir | 2021;57:2003690.

31. Vasarmidi E, Tsitoura E, SpandidosDA, Tzanakis N, Antoniou KM. Pulmonary fibrosis in the aftermath of the COVID-19 era. Exp Ther Med 2020;20:2557-60.
32. Kimura S, Stoicea N, Rosero Britton BR, Shabsigh M, Branstiter A Stahl DL. Preventing ventilator associated lung injury: a perioperative perspective. Front Med (Lausanne) 2016;3:25.

33. Oeckler RA, Hubmayr RD. Ventilator-associated lung injury: a search for better therapeutic targets. Eur Respir J 2007;30: 1216-26.

34. Chambers RC. Role of coagulation cascade proteases in lung repair and fibrosis. Eur Respir J Suppl 2003;44:33s-35s.

35. Erjefalt JS, Sundler F, Persson CG. Eosinophils, neutrophils, and venular gaps in the airway mucosa at epithelial removalrestitution. Am J Respir Crit Care Med 1996;153:1666-74.

36. Wilson MS, Wynn AT. Pulmonary fibrosis: pathogenesis, etiology and regulation. Mucosal Immunol 2009;2:103-21.

37. Ojo AS, Balogun SA, Williams OT, Ojo OS. Pulmonary fibrosis in COVID-19 survivors: predictive factors and risk reduction strategies. Pulm Med 2020;2020:6175964.

38. Wallace WAH, Fitch PM, Simpson AJ, Howie SEM. Inflammationassociated remodelling and fibrosisin the lung - a process and an end point. Int J Exp Pathol 2007;88:103-10.

39. Bian J, Li Z. Angiotensin-converting enzyme 2 (ACE2): SARS-CoV-2 receptor and RAS modulator. Acta Pharm Sin B 2021;11:1-12.

40. Liu Y, Yang Y, Zhang C, Huang F, Wang F, Yuan J. Clinical and biochemical indexes from 2019-nCoV infected patients linked to viral loads and lung injury. Sci China Life Sci 2020;63: 364-74.

41. Wettstein L, Weil T, Conzelmann C, Müller JA, Groß R, Hirschenberger $M$, et al. Alpha-1 Antitrypsin inhibits TMPRSS2 protease activity and SARS-CoV-2 infection. Nat Commun 2021; 12:1726.

42. Vianello A, Braccioni F. Geographical overlap between alpha-1 antitrypsin deficiency and COVID-19 infection in Italy: casual or causal? Arch Bronconeumol 2020;56:609-10.

43. Vianello A, Guarnieri G, Braccioni F, Molena B, Lococo S, Achille A, et al. Correlation between $\alpha$ - 1 antitrypsin deficiency and SARS-CoV-2 infection: epidemiological data and pathogenetic hypotheses. J Clin Med 2021;10:4493.

44. Fehrenbach $\mathrm{H}$. Alveolar epithelial type II cell: defender of the alveolus revisited. Respir Res 2001;2:33-46.

45. Barkauskas CE, Cronce MJ, Rackley CR, Bowie EJ, Keene DR, Stripp BR, et al. Type 2 alveolar cells are stem cells in adult lung. Clin Invest 2013;123:3025-36.

46. Amarante-Mendes GP, Adjemian S, Branco LM, Zanetti LC, Weinlich R, Bortoluci KR. Pattern recognition receptors and the host cell death molecular machinery. Front Immunol 2018;9: 2379.

47. Sardu C, Gambardella J, Morelli MB, Wang X, Marfella R, Santulli G. Hypertension, thrombosis, kidney failure, and diabetes: is COVID-19 an endothelial disease? A comprehensive evaluation of clinical and basic evidence. J Clin Med 2020;9:1417.

48. Cao X. COVID-19: immunopathology and its implications for therapy. Nat Rev Immunol 2020;20:269-70.

49. Hu Z-J, Xu J, Yin JM, Li L, Hou W, Zhang L-L, et al. Lower circulating Interferon-gamma is a risk factor for lung fibrosis in COVID-19 patients. Front Immunol 2020;11:585647.

50. Dreyfuss D, Saumon G. Ventilator-induced lung injury: lessons from experimental studies. Am J RespirCrit Care Med 1998;157: 294-323.

51. Yang Y, Hu L, Xia H, Chen L, Cui S, Wang Y, et al. Resolvin D1 attenuates mechanical stretch-induced pulmonary fibrosis via 
epithelial-mesenchymal transition. Am J Physiol Lung Cell Mol Physiol 2019;316:L1013-24.

52. Yu M, Liu Y, Xu D, Zhang R, Lan L, Xu H. Prediction of the development of pulmonary fibrosis using serial thin-section $\mathrm{CT}$ and clinical features in patients discharged after treatment for COVID-19 pneumonia. Korean J Radiol 2020;21:746-55.

53. Wang JM, Liu W, Chen X, McRae MP, McDevitt JT, Fenyö D. Predictive modeling of morbidity and mortality in patients hospitalized with COVID-19 and its clinical implications: algorithm development and interpretation. J Med Internet Res 2021;23:e29514.

54. Ueland T, Holter JC, Holten AR, Müller KE, Lind A, Bekken GK, et al. Distinct and early increase in circulating MMP-9 in COVID-19 patients with respiratory failure. J Infect 2020;81:e41-3.

55. Guiot J, Moermans C, Henket M, Corhay J-L, Louis R. Blood biomarkers in idiopathic pulmonary fibrosis. Lung 2017;195: 273-80.

56. Xu J, Zheng J, Song P, Zhou Y, Guan S. IL-33/ST2 pathway in a bleomycin-induced pulmonary fibrosis model. Mol Med Rep 2016;14:1704-8.

57. Ragusa R, Basta G, Del Turco S, Caselli C. A possible role for ST2 as prognostic biomarker for COVID-19. Vasc Pharmacol 2021;138: 106857.
58. ZhouM, Yin Z, Xu J, Wang S, Liao T, Wang K, et al. Inflammatory profiles and clinical features of COVID-19 survivors three months after discharge in Wuhan, China. J Infect Dis 2021 Apr 4. https:// doi.org/10.1093/infdis/jiab181 [Epub ahead of print].

59. Ishikawa N, Hattori N, Yokoyama A, Kohno N. Utility of KL-6/MUC1 in the clinical management of interstitial lung diseases. Respir Investig 2012;50:3-13.

60. Kuwana M, Shirai Y, Takeuchi T. Elevated serum Krebs von den Lungen- 6 in early disease predicts subsequent deterioration of pulmonary function in patients with systemic sclerosis and interstitial lung disease. J Rheumatol 2016;43:1825-31.

61. Arnold DT, Donald C, Lyon M, Hamilton FW, Morley AJ, Attwood M, et al. Krebs von den Lungen 6 (KL-6) as a marker for disease severity and persistent radiological abnormalities following COVID-19 infection at12 weeks. PLoS One 2021;16:e0249607.

62. Peng D-H, Luo Y, Huang L-J, Liao F-L, Liu Y-Y, Tang P, et al. Correlation of Krebs von den Lungen- 6 and fibronectin with pulmonary fibrosis in coronavirus disease 2019. Clin Chim Acta 2021;517:48-53.

63. Leeming DJ, Genoves EF, Sand JMB, Rasmussen DGK, Christiansen C, Jenkins G, et al. Can biomarkers of extracellular matrix remodelling and wound healing be used to identify high risk patients infected with SARS-CoV-2?: lessons learned from pulmonary fibrosis. Respir Res 2021;22:38. 